\title{
Highlights of
}

\section{High-Performance Biomimetic Water Channel: The}

\section{Constructive Interplay of Interaction Parameters and Hydrophilic Doping Level}

\author{
Mina Ebrahimi ${ }^{1}$, Masumeh Foroutan ${ }^{1, *}$ \\ ${ }^{1}$ Department of Physical Chemistry, School of Chemistry, College of Science, University of
}

Tehran, Tehran, Iran

The present work introduces a high-performance biomimetic channel mimicked from the hydrophobicity scales of residues in Aquaporin (AQP) pore-lining. We used the molecular dynamics (MD) simulation to inspect the impact of hydrophilic doping level in the biomimetic channel and of the water-wall interaction strength on the water permeability. Accordingly, a unique set of Lennard-Jones (LJ) $\varepsilon$ parameters is discovered for each biomimetic channel with different hydrophilic doping levels, enhancing the water permeation. In biomimetic channels 
simulated with the optimized $\varepsilon$ set, we interestingly obtained a varying pore geometry mimicking the pore analysis results of AQPs. Further investigations on the water transport mechanism proved that tailoring distinct hydrophilic arrays within the hydrophobic backbone allows waterwall vdW energy profile to break the large barrier into multiple reduced ones. The "water jumping" phenomenon also occurs in the middle of the biomimetic channel impelled by two large super-hydrophobic regions on both sides under specific circumstances. In the biomimetic channel, the highest value of water permeability is measured about $(13.67 \pm 0.66) \times 10^{-13} \mathrm{~cm}^{3} / \mathrm{s}$, exhibiting the best mechanism for artificial water channels (AWCs) that water molecules permeate superfast due to the less entrance barrier and weak interaction within the nanotube. Moreover, tuning LJ potential parameters with the doping levels of hydrophilic segments make a general guideline for future study that evolves AWCs design. 